\title{
Quantification of growth asymmetries in developing epithelia
}

\author{
T. Bittig ${ }^{1, a}$, O. Wartlick ${ }^{2, a}$, M. González-Gaitán ${ }^{2, b}$, and F. Jülicher ${ }^{1, c}$ \\ 1 Max Planck Institute for the Physics of Complex Systems, Nöthnitzer Straße 38, 01187 Dresden, Germany \\ 2 Department of Biochemistry and Department of Molecular Biology, Geneva University, Sciences II, Quai Ernest-Ansermet \\ 30, 1211 Geneva 4, Switzerland
}

Received 20 March 2009 and Received in final form 15 July 2009

Published online: 18 September 2009 - (C) EDP Sciences / Società Italiana di Fisica / Springer-Verlag 2009

\begin{abstract}
Many developmental processes of multicellular organisms involve the patterning and growth of two-dimensional tissues, so called epithelia. We have quantified the growth of the wing imaginal disk, which is the precursor of the adult wing, of the fruit fly Drosophila melanogaster. We find that growth follows a simple rule with exponentially decreasing area growth rate. Anisotropies of growth can be precisely determined by comparing experimental results to a continuum theory. Growth anisotropies are to good approximation constant in space and time. They are weak in wild-type wing disks but threefold increased in GFP-Dpp disks in which the morphogen Dpp is overexpressed. Our findings indicate that morphogens such as Dpp control tissue shape via oriented cell divisions that generate anisotropic growth.
\end{abstract}

PACS. 87.18.Fx Multicellular phenomena, biofilms - 87.17.Ee Growth and division - 82.70.Gg Gels and sols

\section{Introduction}

During the development of multicellular organisms from a fertilized egg, tissues form by repeated cell division and attain complex morphologies [1,2]. Fundamental problems are to understand how well-defined shapes and patterns appear and how the final size of the organism is determined. An important model system for the study of the interplay of patterning and growth is the developing wing of the fruit fly Drosophila melanogaster.

The precursor of the adult wing, the so-called wing imaginal disk, is an epithelium or two-dimensional sheet of cells which is closed in spherical topology and forms a double layer $[3,4]$. A schematic representation of the geometry of the wing disk is shown in fig. 1. About 24 hours after fertilization, when the larva hatches, the wing disk contains about 50 cells $[5,6]$. Five days later when the larva enters the pupal stage, the wing disk has grown during 10 cell generations to about 50000 cells [5,7] and attained a characteristic shape and size. Patterns of gene expression are established with the help of morphogens. Morphogens are secreted in localized sources (see fig. 1). They spread in the tissue to form graded concentration profiles by the interplay of unbiased spreading and degradation [8-12]. A key morphogen in the wing disk is Decapentaplegic

\footnotetext{
a These authors contributed equally to this work.

b e-mail: marcos.gonzalez@unige.ch

c e-mail: julicher@pks.mpg.de
}

(Dpp) $[13,14]$. It is secreted along a linear source region defined by the boundary separating anterior from posterior cells [15] (see fig. 1). Similarly, a source of the morphogen Wingless $(\mathrm{Wg}$ ) exists at the dorso-ventral compartment boundary. In addition to providing positional information to cells in the epithelium, morphogens such as Dpp are also involved in the regulation of growth and thus cell division [12,13,16-22]. Several models have been proposed to explain how graded Dpp signaling could mediate spatially homogeneous proliferation in the wing imaginal disk. One hypothesis is that cells interpret the slope of the gradient $[18,19]$. Alternatively, cells could read absolute Dpp concentrations, but mechanical stresses or other feedback could make growth regulation spatially uniform [20-22]. Quantitative evidence for either model is lacking, and thus the principles underlying growth control are still under debate. Here we present a quantitative study of growth in developing epithelia and show that in the wing imaginal disk, Dpp influences growth anisotropies.

From the point of view of physics, growing tissues are a form of active soft matter [23-26] which is dynamically remodeled by cell division and cell death. The resulting pattern of cell packing and shape of the multicellular system emerges as a collective behavior of many cells and their interactions. Cell division and its stochastic properties plays a crucial role in controlling the morphology of the resulting tissue. Different physical approaches have been used to describe tissue dynamics and reorganization at different length scales [27-33]. When discussing the 


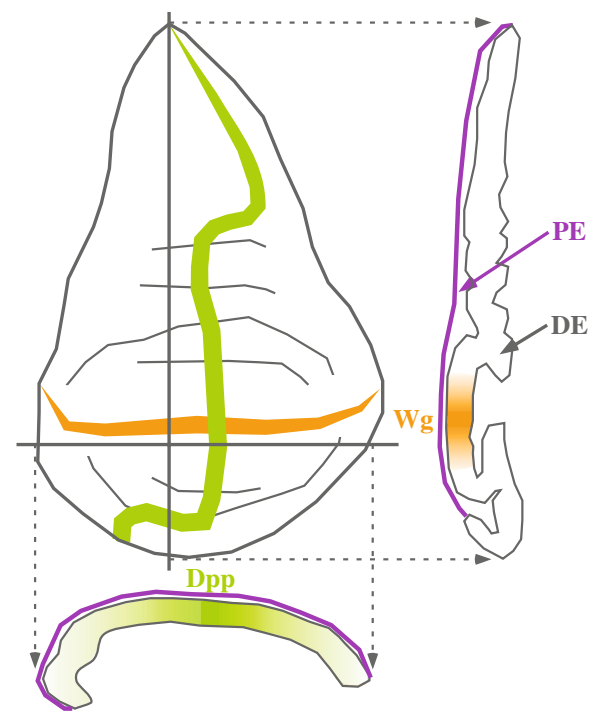

Fig. 1. Schematic view of a third instar wing imaginal disk with lateral and transverse sections (arrows). The disk is oriented with anterior to the left. The columnar disk epithelium (DE) is covered on the apical side by a squamous epithelium of peripodial cells (PE). The morphogens Dpp and $\mathrm{Wg}$ are expressed in perpendicular stripes in the center of the disk and form concentration gradients perpendicular to their axes of expression.

mechanics and dynamics of tissues during development, a key question concerns the effective material properties that are relevant for a tissue on scales involving many cells. Individual cells behave as elastic bodies, however in a tissue cells can rearrange and neighborship relations can change. This implies that stresses in the tissue may relax as cell packings are remodeled. A simplified description of cell packings represents cells as elastic objects. They are subject to friction forces when cells slide relative to each other. In such a description, we have shown that at large scales, a hydrodynamic limit exists in which a growing tissue can be described as an active anisotropic visco-elastic fluid [33]. This limit results in particular from stress relaxation due to cell rearrangements triggered by cell division. In addition, it was shown that anisotropies in the system such as those imposed by morphogen gradients or planar cell polarity $[34,35]$ can, via oriented cell division, induce anisotropic stresses and flow fields [33]. Here oriented cell division can refer to both a preferred orientation of the mitotic spindle during cell division [36] or to a reorientation of the daughter cells after cell division.

In this paper, we quantify the shape changes as a function of time of the wing imaginal disk. We compare two situations: i) wild-type wing disks (WT disks) and ii) wing disks in which Dpp labelled by Green Fluorescent Protein (GFP) is expressed in addition to the endogenous Dpp (GFP-Dpp disks). In the second case, Dpp is expressed at higher levels. We study the average growth rate as well as the anisotropy of growth and compare our results to a hydrodynamic theory. We show that the observed shape changes during time correspond to a homogeneous and
A
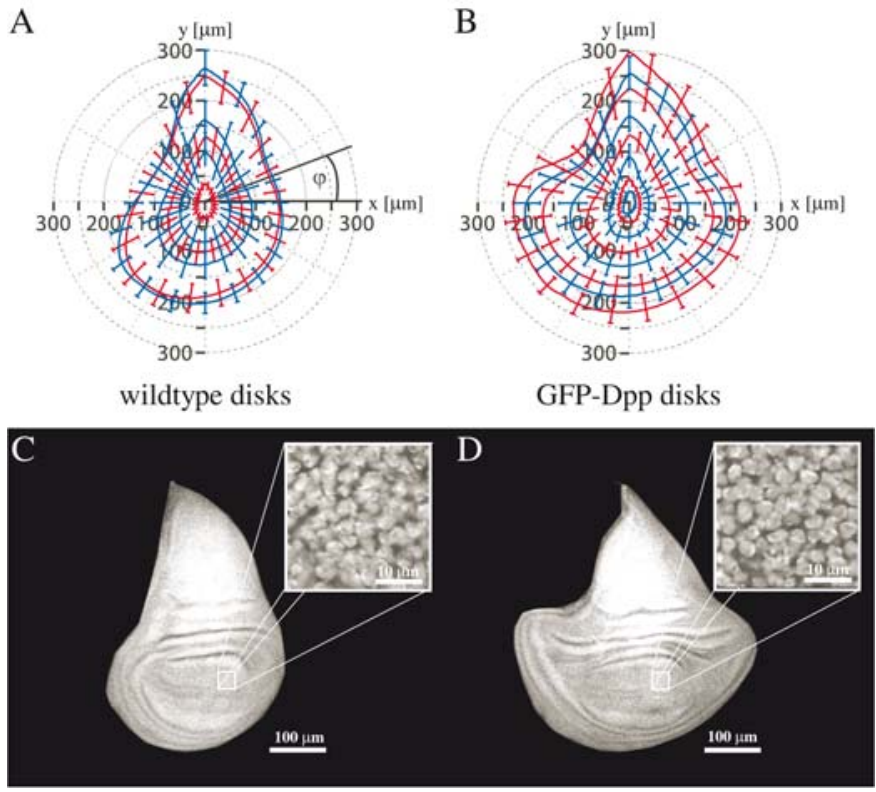

Fig. 2. Average contours $R(\varphi)$ of wing disks at different times during larval development. (A) Wild-type (WT) disks at $36 \mathrm{~h}$, $48 \mathrm{~h}, 60 \mathrm{~h}, 69 \mathrm{~h}, 84 \mathrm{~h}$ and $96 \mathrm{~h}$ after hatching. (B) GFP-Dpp disks at $24 \mathrm{~h}, 36 \mathrm{~h}, 48 \mathrm{~h}, 60 \mathrm{~h}, 72 \mathrm{~h}, 84 \mathrm{~h}, 96 \mathrm{~h}$ and $120 \mathrm{~h}$ after hatching. The origin of the coordinate system is the center of the area of the disks. The error bars given are standard deviations. (C) Representative WT disk at the end of larval development (96 h after hatching), stained with DAPI. The inset shows nuclei on one $z$-frame at higher magnification. (D) Representative GFP-Dpp disk at the end of development $(120 \mathrm{~h}$ after hatching for GFP-Dpp larvae).

anisotropic growth, the anisotropy of which depends on the Dpp level. This implies that both size and shape of the tissue are controlled via oriented cell division.

\section{Quantification of tissue shapes during growth}

Wing disks were dissected from larvae at different times after hatching, see appendix A. Shapes of wing disks of different ages are shown in fig. 2. The center of area of all contours is located at the origin of the $x y$-plane. The contours $R(\varphi)$, where $R$ is the distance of the contour line from the origin and $\varphi$ is the polar angle, are oriented such that the $y$-axis corresponds to the largest diameter. While at early times the shapes of WT (fig. 2A) and GFP-Dpp disks (fig. 2B) are similar, they differ visibly at later times. Inspection of fig. 2 suggests that the GFP-Dpp disks grow more anisotropically as compared to WT disks.

Growth of the disks can be quantified by determining the area $A$ as a function of time. The area as a function of time during growth is shown in fig. $3 \mathrm{~A}$. We fit this data by a function which describes the simplest possible scenario of finite growth. Since growth stops eventually, the area growth rate $k(t)$ changes with time and vanishes for long times. We find that a growth rate which is initially (at 
A

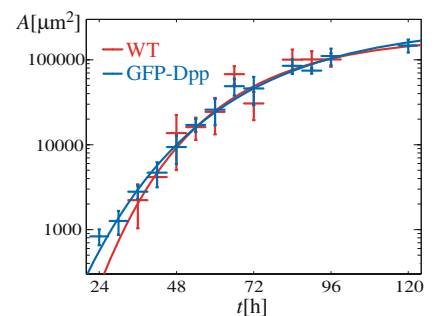

C

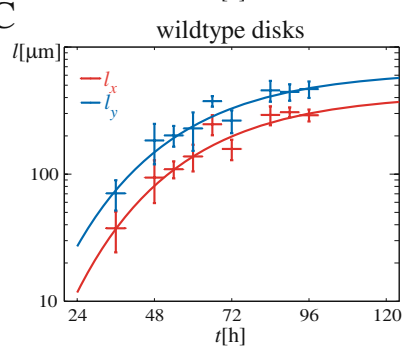

B

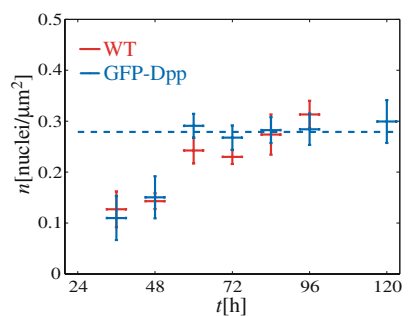

$\mathrm{D}$

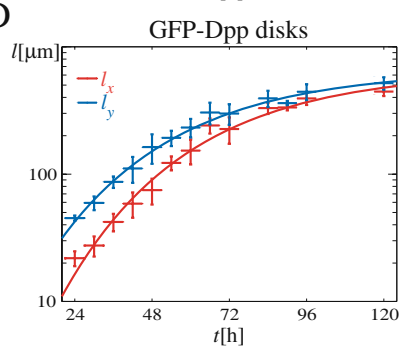

Fig. 3. (Colour on-line) Area growth, cell density and linear growth. (A) Growth of the area $A$ for WT (red) and GFP-Dpp disks (blue). (B) Nuclear density of the disk epithelium (DE, see fig. 1 and appendix A) of WT (red) and GFP-Dpp disks (blue) as a measure of cell density. (C) Disk diameters $l_{x}$ and $l_{y}$ along $x$ - and $y$-axes for WT disks. (D) Diameters $l_{x}$ and $l_{y}$ for GFP-Dpp disks. Each data point is the average of about 10 disks (121 disks in total for each genotype). The solid lines in (A) are fits of eq. (2) and in (C, D) of eq. (3) to the data, see main text and table 1 . The dashed line in (B) indicates that the nuclear density stays approximately constant after $60 \mathrm{~h}$.

$\left.t=t_{0}\right)$ given by $k^{(0)}$ and which then decays exponentially with a single relaxation time $\tau$, can quantitatively account for the data. We therefore write

$$
k(t)=\frac{\dot{A}}{A}=k^{(0)} e^{-\left(t-t_{0}\right) / \tau},
$$

where the dot denotes a time derivative. The corresponding time dependence of the area is

$$
A(t)=A^{(0)} \exp \left(k^{(0)} \tau\left(1-e^{-\left(t-t_{0}\right) / \tau}\right)\right),
$$

where $A^{(0)}$ is the initial area at $t=t_{0}$. This function is fit to the data in fig. $3 \mathrm{~A}$.

The area attained for large times is $A^{(\mathrm{f})}=$ $A^{(0)} \exp \left(k^{(0)} \tau\right)$. We fit eq. (2) to the data, using $t_{0}, k^{(0)}$ and $\tau$ as fit parameters. As initial area, we use $A^{(0)}=$ $264 \mu \mathrm{m}^{2}$ (estimated from [6]). From the fit displayed in fig. 3A, we obtain for WT disks $k^{(0)}=0.22 \pm 0.02 \mathrm{~h}^{-1}$ and $\tau=29.7 \pm 4.0 \mathrm{~h}$ and for GFP-Dpp disks $k^{(0)}=$ $0.18 \pm 0.01 \mathrm{~h}^{-1}$ and $\tau=38.1 \pm 2.2 \mathrm{~h}$. The area increase $A^{(\mathrm{f})} / A^{(0)}=\exp \left(k^{(0)} \tau\right)$ is thus 714 for WT and 997 for GFP-Dpp disks, see table 1 .

In order to quantify the anisotropy of growth, we measured the linear growth rates $k_{x}$ and $k_{y}$ along two perpendicular axes. We define the diameters $l_{x}(t)$ and $l_{y}(t)$ of the disk contours along the $x$ - and $y$-axis, respectively. The time dependence of linear growth along the $x$ - and $y$-axes is shown in fig. $3 \mathrm{C}$ and D. In WT disks, both diameters start at different values but grow at similar rates.

In the case of GFP-Dpp disks, where Dpp is overexpressed, the length $l_{x}(t)$ grows significantly faster than the length $l_{y}(t)$. For both WT and GFP-Dpp disks, the growth of the lengths $l_{x}(t)$ and $l_{y}(t)$ is again well described by the functions

$$
l_{x, y}(t)=l_{x, y}^{(0)} \exp \left(k_{x, y}^{(0)} \tau_{x, y}\left(1-e^{-\left(t-t_{0}\right) / \tau_{x, y}}\right)\right),
$$

where $l_{x, y}^{(0)}$ is the tissue diameter at the initial time $t_{0}$ and $\tau_{x, y}$ is the characteristic time of growth in $x$ - and $y$ direction, respectively. We define the linear growth rates $k_{x}(t)=\dot{l}_{x}(t) / l_{x}(t)$ and $k_{y}=\dot{l}_{y}(t) / l_{y}(t)$ along the $x$ - and $y$-axis, respectively. Here, the parameters $k_{x, y}^{(0)}$ denote the initial growth rates and

$$
k_{x, y}(t)=k_{x, y}^{(0)} e^{-\left(t-t_{0}\right) / \tau_{x, y}} .
$$

We fit eq. (3) to the data, using $l_{x, y}^{(0)}, k_{x, y}^{(0)}$ and $\tau_{x, y}$ as fit parameters. We use the value of $t_{0}$ obtained from the analysis of the area growth, see table 1 . We find that for both WT and GFP-Dpp disks the characteristic time constants $\tau_{x}$ and $\tau_{y}$ describing the growth along the two main axes are the same $\left(\tau_{x}=31.9 \pm 4.2 \mathrm{~h}\right.$ and $\tau_{y}=30.3 \pm$ $3.9 \mathrm{~h}$ for WT disks and $\tau_{x}=39.5 \pm 2.5 \mathrm{~h}$ and $\tau_{y}=36.8 \pm$ $2.1 \mathrm{~h}$ for GFP-Dpp disks). We thus also perform fits using a single time constant $\tilde{\tau}$. The characteristic time $\tilde{\tau}$ during which growth occurs is larger for GFP-Dpp disks than for WT disks (see table 1), consistent with the observed longer growth period of GFP-Dpp disks (see fig. 2). We find that more growth occurs along the $x$-direction as compared to the $y$-direction (see values of $l_{x}^{(f)} / l_{x}^{(0)}$ as compared to $l_{y}^{(\mathrm{f})} / l_{y}^{(0)}$ in table 1). Furthermore, this growth anisotropy is larger for GFP-Dpp disks as compared to WT disks.

A quantity that characterizes the anisotropy of growth is the rate $k_{1}(t)=\left(k_{x}(t)-k_{y}(t)\right) / 2$ that can be compared to the average growth rate $k_{0}(t)=\left(k_{x}(t)+k_{y}(t)\right) / 2$. From eq. (4) it follows that

$$
k_{0,1}(t)=\frac{k_{x}^{(0)} \pm k_{y}^{(0)}}{2} e^{-\left(t-t_{0}\right) / \tilde{\tau}},
$$

where the plus sign holds for $k_{0}$ and the minus sign for $k_{1}$. The dimensionless ratio $\epsilon=k_{1} / k_{0}$ characterizing the anisotropy of growth is independent of time, $\epsilon=\left(k_{x}^{(0)}+\right.$ $\left.k_{y}^{(0)}\right) /\left(k_{x}^{(0)}-k_{y}^{(0)}\right)$. On the basis of our data analysis (see table 1 ), we find $\epsilon=0.063 \pm 0.067$ for WT disks and $\epsilon=0.143 \pm 0.033$ for GFP-Dpp disks. We thus find that the anisotropy depends on the Dpp expression level. Note that the errors in determining $\epsilon$ are significant and the analysis cannot rule out that wild-type disks grow isotropically. We now compare our data to a continuum description of growth which allows us to quantify growth anisotropies more reliably.

\section{Continuum theory of anisotropic growth}

In a continuum theory, the two-dimensional tissue is characterized by a cell number density $\rho$ and a cellular flow 
Table 1. Parameters describing area and linear growth of WT and GFP-Dpp wing disks, see fig. 3. For all quantities, the optimized fit parameters and standard errors obtained by least square fits of eqs. (2) and (3) to the measured data are displayed. Additionally, the ratios $A^{(\mathrm{f})} / A^{(0)}=\exp \left(k^{(0)} \tau\right)$ and $l_{x, y}^{(\mathrm{f})} / l_{x, y}^{(0)}=\exp \left(k_{x, y}^{(0)} \tilde{\tau}\right)$ are shown.

\begin{tabular}{lcccccc}
\hline \hline & $t_{0}(\mathrm{~h})$ & $A^{(\mathrm{f})} / A^{(0)}$ & $l_{x}^{(0)}(\mu \mathrm{m})$ & $l_{x}^{(\mathrm{f})} / l_{x}^{(0)}$ & $l_{y}^{(0)}(\mu \mathrm{m})$ & $l_{y}^{(\mathrm{f})} / l_{y}^{(0)}$ \\
\hline WT & $24.9 \pm 1.5$ & 714 & $13.0 \pm 1.5$ & 33 & $29.6 \pm 3.2$ & 22 \\
GFP-Dpp & $19.6 \pm 0.8$ & 997 & $10.5 \pm 0.8$ & 62 & $30.3 \pm 2.0$ & 22 \\
\hline \hline & \multicolumn{7}{c}{$k^{(0)}\left(\mathrm{h}^{-1}\right)$} & $\tau(\mathrm{h})$ & $k_{x}^{(0)}\left(\mathrm{h}^{-1}\right)$ & $k_{y}^{(0)}\left(\mathrm{h}^{-1}\right)$ & $\tilde{\tau}(\mathrm{h})$ \\
\hline WT & $0.22 \pm 0.02$ & $29.7 \pm 4.0$ & $0.112 \pm 0.011$ & $0.099 \pm 0.010$ & $31.2 \pm 2.9$ \\
GFP-Dpp & $0.18 \pm 0.01$ & $38.1 \pm 2.2$ & $0.107 \pm 0.005$ & $0.080 \pm 0.004$ & $38.5 \pm 1.7$ \\
\hline \hline
\end{tabular}

field $\mathbf{v}=\left(v_{x}, v_{y}\right)$ which obey the balance equation

$$
\partial_{t} \rho+\nabla \cdot(\rho \mathbf{v})=k \rho
$$

Here $k=k_{g}-k_{a}$ is the effective cell doubling rate and $k_{g}$ and $k_{a}$ denote the rates of cell division and cell death, respectively. We consider in the following the simple case where the cell area is incompressible and the cell density is constant, i.e. $\rho=\rho_{0}$ and $\nabla \cdot \mathbf{v}=k$. This implies that the effective cell doubling rate equals the local area growth rate. This simplification is motivated by the observation that in both wild-type and GFP-Dpp disks the increase in cell density during development is small compared to the overall increase in area (see fig. 3A, B).

Equation (6) is complemented by a constitutive material relation and a force balance condition. In the viscous limit, which we consider here for simplicity, the constitutive relation links the symmetric velocity gradient tensor $u_{\alpha \beta}=(1 / 2)\left(\partial_{\alpha} v_{\beta}+\partial_{\beta} v_{\alpha}\right)$ to the stress tensor $\sigma_{\alpha \beta}$, with $\alpha$ and $\beta=x, y$. It has been suggested that anisotropic growth is a consequence of anisotropic active stresses generated in the growing tissue. In particular, oriented cell division in general gives rise to such anisotropic stresses [33]. Active anisotropic stresses due to cell division also enter the constitutive material relation which reads

$$
2 \eta \tilde{u}_{\alpha \beta}=\tilde{\sigma}_{\alpha \beta}+\mu k_{\mathrm{g}} \tilde{q}_{\alpha \beta} .
$$

Here $\tilde{u}_{\alpha \beta}=u_{\alpha \beta}-u_{\gamma \gamma} \delta_{\alpha \beta}$ and $\tilde{\sigma}_{\alpha \beta}=\sigma_{\alpha \beta}-\sigma_{\gamma \gamma} \delta_{\alpha \beta}$ are the traceless parts of the velocity gradient and stress tensors, respectively. The anisotropy is characterized by the traceless tensor $\tilde{q}_{\alpha \beta}=p_{\alpha} p_{\beta}-(1 / 2) \delta_{\alpha \beta}$ where the unit vector $\mathbf{p}$ defines the preferred axis of cell division. The tissue viscosity is denoted $\eta$. Note that in general in an anisotropic system the viscosity is also anisotropic [26]. Here, we neglect such effects. The coefficient $\mu$, which has dimension of viscosity, characterizes the active anisotropic stress induced by oriented cell division. Note that in general $\mu$ depends on cell density. For small density, $\mu$ is expected to be proportional to cell density, $\mu \sim \rho$.

The force balance reads $\partial_{\beta} \sigma_{\alpha \beta}-\partial_{\alpha} P=0$, where the pressure $P$ plays the role of a Lagrange multiplier to impose the incompressibility constraint $u_{\gamma \gamma}=k$. These equations together with the boundary conditions $\sigma_{n n}=$ $P-P_{\text {ext }}$ and $\sigma_{n t}=0$, where $P_{\text {ext }}$ is an external pressure,

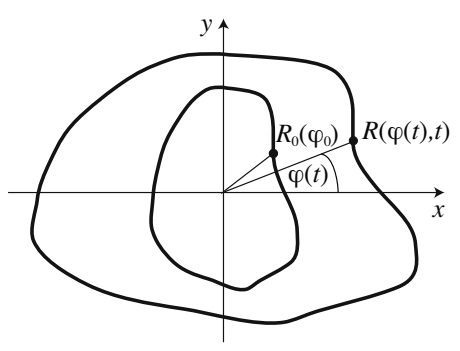

Fig. 4. Shape change of an epithelium boundary due to anisotropic tissue growth. The tissue boundary is shown at the initial time $t_{0}$ and the later time $t$. The preferred orientation of the cell division axis is $\mathbf{p}=\mathbf{e}_{x}$. The position of a cell which is situated at the tissue boundary is marked by a dot and described by the radial distances $R_{0}\left(\varphi_{0}\right)$ and $R(\varphi(t), t)$. The polar angle describing the position of the marked cell changes from the initial angle $\varphi_{0}=\varphi\left(t_{0}\right)$ to the later angle $\varphi(t)$.

determine the flow field. For homogeneous growth, with values of $k, \eta$ and $\mu$ that are independent of position, the velocity field reads

$$
\mathbf{v}=\left(\begin{array}{c}
\left(k_{0}+k_{1}\right) x \\
\left(k_{0}-k_{1}\right) y
\end{array}\right) .
$$

Here $k_{0}=\left(k_{\mathrm{g}}-k_{\mathrm{a}}\right) / 2$ and $k_{1}=k_{\mathrm{g}} \mu /(4 \eta)$. Note that this form of the flow field is independent of the shape of the system but applies only inside the contour line that defines the tissue boundary $R(\varphi, t)$.

\section{Shape changes and angle-dependent growth rates}

We consider the effects of anisotropic growth on the time evolution of the tissue boundary $R(\varphi, t)$, see fig. 4. A cell on the boundary at time $t=t_{0}$ with coordinates $X_{0}=R_{0} \cos \varphi_{0}, Y_{0}=R_{0} \sin \varphi_{0}$ moves as a consequence of growth along a trajectory $X(t), Y(t)$ with $\mathrm{d} X / \mathrm{d} t=$ $v_{x}(X, Y)$ and $\mathrm{d} Y / \mathrm{d} t=v_{y}(X, Y)$. In polar coordinates,

$$
\left(\begin{array}{l}
X(t) \\
Y(t)
\end{array}\right)=R(\varphi(t), t)\left(\begin{array}{c}
\cos (\varphi(t)) \\
\sin (\varphi(t))
\end{array}\right),
$$


A
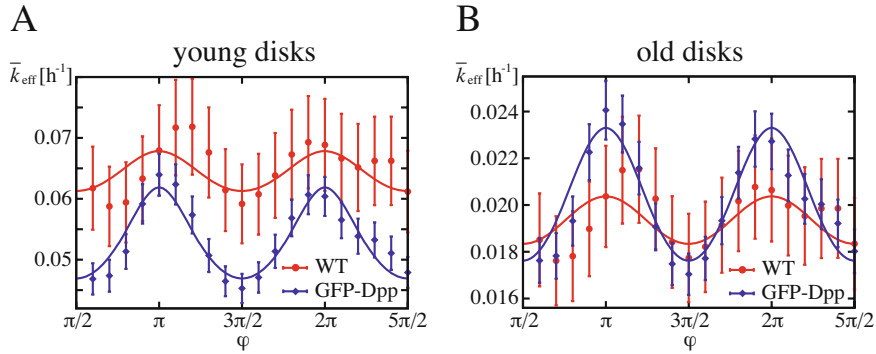

Fig. 5. Averaged effective growth rates for young and old wing disks as functions of the polar angle $\varphi$. The rates for both WT and GFP-Dpp disks are shown. The dots and corresponding error bars represent $\bar{k}_{\text {eff }}$ determined from the experimental data, see text. The solid lines represent the theoretical curves (12) of $\bar{k}_{\text {eff }}(\varphi)$ in which we inserted the growth rates $k_{0}(t)$ and $k_{1}(t)$ which were quantified by our analysis of the measured lengths $l_{x, y}(t)$, see fig. 3 and eq. (5). The time interval for the young disks (A) was chosen as [24 h, $60 \mathrm{~h}]$, and for the old disks we have chosen the time interval as $[60 \mathrm{~h}, 96 \mathrm{~h}]$ for both WT and GFP-Dpp disks.

where $\tan \varphi(t)=Y(t) / X(t)$. From the cell trajectories $X(t)=X_{0} \exp \left(\int_{t_{0}}^{t}\left(k_{0}+k_{1}\right) \mathrm{d} t^{\prime}\right), Y(t)=Y_{0} \exp \left(\int_{t_{0}}^{t}\left(k_{0}-\right.\right.$ $\left.\left.k_{1}\right) \mathrm{d} t^{\prime}\right)$, it follows that $\tan \varphi(t)=\tan \varphi_{0} \exp \left(-2 \int_{t_{0}}^{t} k_{1} \mathrm{~d} t^{\prime}\right)$. We obtain an equation for the contour of the tissue boundary

$$
\begin{aligned}
R(\varphi, t)= & R_{0}(\psi) e^{\int_{t_{0}}^{t} k_{0} \mathrm{~d} t^{\prime}} \\
& \times\left(\sin ^{2} \varphi e^{2 \int_{t_{0}}^{t} k_{1} \mathrm{~d} t^{\prime}}+\cos ^{2} \varphi e^{-2 \int_{t_{0}}^{t} k_{1} \mathrm{~d} t^{\prime}}\right)^{-1 / 2}
\end{aligned}
$$

where $\psi(\varphi, t)=\arctan \left(\tan (\varphi) \exp \left(2 \int_{t_{0}}^{t} k_{1} \mathrm{~d} t^{\prime}\right)\right)$. The initial shape is $R_{0}(\varphi)$ and $\varphi\left(t_{0}\right)=\varphi_{0}$.

We define the effective growth rate at an angle $\varphi$ by $k_{\text {eff }}(\varphi)=\dot{R}(\varphi) / R(\varphi)$. Using eq. (10), we find

$$
k_{\mathrm{eff}}(\varphi, t) \approx k_{0}-k_{1} \frac{\sin ^{2} \varphi e^{2 \int_{t_{0}}^{t} k_{1} \mathrm{~d} t^{\prime}}-\cos ^{2} \varphi e^{-2 \int_{t_{0}}^{t} k_{1} \mathrm{~d} t^{\prime}}}{\sin ^{2} \varphi e^{2 \int_{t_{0}}^{t} k_{1} \mathrm{~d} t^{\prime}}+\cos ^{2} \varphi e^{-2 \int_{t_{0}}^{t} k_{1} \mathrm{~d} t^{\prime}}},
$$

where we used the approximation $\varphi(t) \approx \varphi_{0}$. Equation (11) shows that the effective growth rate along an angle $\varphi$ is determined by the average growth rate $k_{0}(t)$ and modulated by the anisotropic part of the growth rate $k_{1}(t)$. The time average $\bar{k}_{\text {eff }}(\varphi)=\frac{1}{t_{2}-t_{1}} \int_{t_{1}}^{t_{2}} k_{\text {eff }}(\varphi, t) \mathrm{d} t$ of the effective growth rate during a time interval $\left[t_{1}, t_{2}\right]$ is thus given by

$$
\begin{aligned}
\bar{k}_{\mathrm{eff}}(\varphi) \approx & \frac{1}{t_{2}-t_{1}}\left[\int_{t_{1}}^{t_{2}} k_{0} \mathrm{~d} t\right. \\
& \left.-\frac{1}{2} \ln \left(\sin ^{2} \varphi e^{2 \epsilon \int_{t_{1}}^{t_{2}} k_{0} \mathrm{~d} t}+\cos ^{2} \varphi e^{-2 \epsilon \int_{t_{1}}^{t_{2}} k_{0} \mathrm{~d} t}\right)\right],
\end{aligned}
$$

where we used $k_{1}=\epsilon k_{0}$.
We compare eq. (12) to the observed angular distribution of effective growth rates in the wing disk, see appendix B. Figure 5 shows the average effective growth rates $\bar{k}_{\text {eff }}$ during the time intervals $[24 \mathrm{~h}, 60 \mathrm{~h}]$ after hatching (young disks) and [60 h, $96 \mathrm{~h}]$ after hatching (old disks). This choice of time intervals yields a good statistics for our analysis of the 121 wing disks. Consistent with a decrease in the growth rate, fig. 5 indicates that the average growth rate is larger in young disks (see fig. 5A) than in old disks (see fig. 5B) for both WT and GFP-Dpp disks. In both cases (WT and GFP-Dpp disks), the angular distribution of the growth rates has a maximum close to the $x$-axis (corresponding to the angles $\varphi=\pi, 2 \pi$ ) and a minimum near the $y$-axis (corresponding to the angles $\varphi=\pi / 2,3 \pi / 2)$.

In order to quantify the anisotropy of growth, we fit eq. (12) to the angular distribution of time-averaged effective growth rates using the growth rate $k_{0}(t)$ described by eq. (5) with the parameters given in table 1 . The anisotropy of growth $\epsilon$, which characterizes the amplitude of the distribution of effective growth rates, is used as a fit parameter. For disks of a given type (WT or GFPDpp), the growth anisotropy $\epsilon$ is the same for young and old disks, indicating that $\epsilon$ is time-independent. We find $\epsilon=0.051 \pm 0.004$ for WT disks and $\epsilon=0.138 \pm 0.003$ for GFP-Dpp disks. Thus the anisotropy of growth $\epsilon$ can be determined very precisely by the analysis based on our continuum theory of growth.

\section{Discussion}

We have quantitatively analyzed the dynamics and anisotropies of growth of the wing imaginal disk of the fruit fly Drosophila melanogaster during development. We have shown that the area growth exhibits a simple behavior with exponentially decaying growth rate $k$, see eq. (1). The growth rate is maximal at early times $\left(k^{(0)} \simeq 0.2 \mathrm{~h}^{-1}\right)$ and decays exponentially in a characteristic time $\tau$ of the order of 35 hours. During growth the area increases by a factor of about 1000 , and the corresponding cell generation number $n=k^{(0)} \tau / \ln 2$ is about 10 .

The growth of the wing disk is anisotropic. Growth anisotropies can be quantified very precisely on the basis of our continuum description of growth. We quantify the angular growth rate $k_{\mathrm{eff}}(\varphi)$ which exhibits a sinusoidal modulation around the value $k / 2$, see fig. 5 . Using the continuum theory, the data can be fit with the anisotropy $\epsilon$ being the single fit parameter. This growth anisotropy can be related to two axes established during development in the tissue. One axis is characterized by the antereo-posterior $(\mathrm{A} / \mathrm{P})$ compartment boundary which is a sharp and smooth interface dividing the wing disk into two parts [37]. This boundary plays the role of an organizing center for patterning and growth. A narrow line of cells along this compartment boundary expresses the morphogen Dpp which spreads into the tissue and forms graded concentration profiles which decay in the direction perpendicular to the A/P boundary [15]. Interestingly, we find that the axis along which growth is weakest coincides 
with the axis defined by the $\mathrm{A} / \mathrm{P}$ compartment boundary. The direction in which growth is strongest is the direction in which Dpp forms a gradient. This finding indicates that the Dpp gradient controls growth anisotropies, which is interesting in view of earlier suggestions that Dpp gradients could control growth $[18,19]$. Our observations also show that the axis of growth anisotropy remains fixed with respect to the $\mathrm{A} / \mathrm{P}$ compartment boundary. This implies that the axis of cell division is controlled by the orientation of the compartment boundaries. Since the dominant cell division axis remains stable with respect to the compartment boundaries, we do not need to describe cell polarity as a separate dynamic variable.

We find significant differences in growth anisotropy when comparing wild-type and GFP-Dpp wing disks. In wild-type disks this anisotropy is weak with $\epsilon \simeq 0.05$. The GFP-Dpp disks in which both endogenous and GFP-Dpp are produced and functional Dpp thus occurs in higher levels, the growth anisotropy is increased threefold. Furthermore, GFP-Dpp disks exhibit more area growth and grow for a longer time at a slightly smaller rate as compared to wild type.

In our analysis, we have ignored the three-dimensional structure of the wing disk epithelium. Indeed, it is known that the fully grown epithelium exhibits folds along lines approximately parallel to the $x$-axis [4]. In our experiments, we observe that folds begin to form at about $80 \mathrm{~h}$ after hatching. Such folds could influence the apparent area growth because the projected area discussed here can differ slightly from the total area of the epithelium. Since the observed growth anisotropy is constant in time and does not change after $80 \mathrm{~h}$ when folds appear, we assume the effect of folds on growth to be negligible. However, a quantification of the full three-dimensional structural changes during growth remains a challenge for future work.

Since GFP-Dpp wing disks differ from WT disks both in the duration of growth and in growth anisotropy, our findings provide strong evidence that Dpp is involved in the control of both size and shape of the wing disk by controlling the area growth and growth anisotropies. Dpp overexpression as in GFP-Dpp disks has pronounced effects on growth anisotropies. A natural interpretation of this finding is that the Dpp gradient direction controls the axis of cell divisions. Oriented cell division has indeed been observed experimentally [36]. A theoretical study of the effects of oriented cell division has demonstrated that this leads effectively to anisotropic growth as described by the continuum theory [33]. Experimental studies of cell divisions on micropatterned substrates reveal that cortical cues control the cell division axis via rotations of the mitotic spindle [38]. We speculate that the Dpp gradient could set up cortical anisotropies that bias cell division orientation and thus control tissue shape.

We thank Ani Kicheva and Peer Mumcu for stimulating discussions. Ortrud Wartlick and Marcos González-Gaitán were supported by the VW Stiftung, an FP6 Strep (ONCASYM), SNF, SystemsX (LipidX), HFSP and an ERC senior grant.

\section{Appendix A. Materials and methods}

Genotypes used: dpp-Gal4/UAS-GFP-Dpp. dpp-Gal4 is described in Flybase; UAS-GFP-Dpp has been described previously [39]. Six hour egg collections were grown at $25^{\circ} \mathrm{C}$ and larvae were dissected at the indicated times after hatching (see fig. 3). Wing disks were fixed with $4 \%$ Paraformaldehyde, stained with DAPI, and imaged with a $10 \times$ objective at a Zeiss confocal microscope.

To quantify the nuclear density, a $z$-stack through the whole disk was taken with a $60 \times$ objective. A $z$-stack is necessary because nuclei of the disk epithelium (DE, fig. 1) are not located on one $z$-frame only. All nuclei in the DE were counted. Nuclei of the peripodial epithelium (PE, fig. 1) can be reliably excluded because they are more widely spaced (in all but the youngest disks the PE is a squamous epithelium) and separated from the nuclei of the DE by a lumen (in all disks). The nuclear density of the DE was then calculated as (number of nuclei)/(area bounding the $z$-stack).

\section{Appendix B. Quantification of averaged effective growth rates in the wing disk}

We discuss the quantification of the effective growth rates $k_{\text {eff }}\left(\varphi_{m}\right)=\dot{R}\left(\varphi_{m}\right) / R\left(\varphi_{m}\right)$ in the wing disk for the angles $\varphi_{m}=m 2 \pi / 400(m=0,1, \ldots, 399)$. Similar to our analysis of the diameters $l_{x}$ and $l_{y}$ (see eq. (3)), we fit the function $R\left(\varphi_{m}, t\right)=R_{0}\left(\varphi_{m}\right) \exp \left(k_{\text {eff }}^{(0)}\left(\varphi_{m}\right) \tilde{\tau}\left[1-e^{-\left(t-t_{0}\right) / \tilde{\tau}}\right]\right)$ to the observed radial positions $R\left(\varphi_{m}, t\right)$ at each angle $\varphi_{m}$. We choose $t_{0}=20 \mathrm{~h}$ and $\tilde{\tau}$ from table 1 and use $R_{0}\left(\varphi_{m}\right)$ and $k_{\text {eff }}^{(0)}\left(\varphi_{m}\right)$ as fit parameters. The effective growth rate averaged during the time interval $\left[t_{1}, t_{2}\right]$

$$
\bar{k}_{\text {eff }}\left(\varphi_{m}\right)=\frac{1}{t_{2}-t_{1}} \int_{t_{1}}^{t_{2}} k_{\text {eff }} \mathrm{d} t=\frac{1}{t_{2}-t_{1}} \ln \frac{R\left(t_{2}\right)}{R\left(t_{1}\right)}
$$

is thus determined by $\bar{k}_{\text {eff }}\left(\varphi_{m}\right)=\frac{k_{\text {eff }}^{(0)}\left(\varphi_{m}\right) \tilde{\tau}}{t_{\mathrm{f}}-t_{0}}\left(e^{-\left(t_{1}-t_{0}\right) / \tilde{\tau}}-\right.$ $\left.e^{-\left(t_{2}-t_{0}\right) / \tilde{\tau}}\right)$.

\section{References}

1. L. Wolpert, R. Beddington, T. Jessell, P. Lawrence, E. Meyerowitz, J. Smith, Principles of Development (Oxford University Press, Oxford, 2002) 2nd edition.

2. T. Lecuit, L. Le Goff, Nature 450, 189 (2007).

3. S.M. Cohen, in The Development of Drosophila melanogaster, edited by M. Bate, A.M. Arias, Vol. II (Cold Spring Harbor Press, Cold Spring Harbor, New York, 1993).

4. D. Fristrom, J.W. Fristrom, in The Development of Drosophila melanogaster, edited by M. Bate, A.M. Arias, Vol. II (Cold Spring Harbor Press, Cold Spring Harbor, New York, 1993).

5. A. García-Bellido, J.R. Merriam, Dev. Biol. 24, 61 (1971).

6. M.M. Madhavan, H.A. Schneiderman, Roux's Arch. Dev. Biol. 183, 269 (1977). 
7. M.J. Fain, B. Stevens, Dev. Biol. 92, 247 (1982).

8. L. Wolpert, J. Theor. Biol. 25, 1 (1969).

9. M. Strigini, S.M. Cohen, Semin. Cell Dev. Biol. 10, 335 (1999).

10. A. Eldar, R. Dorfman, D. Weiss, H. Ashe, B.Z. Shilo, N. Barkai, Nature 419, 304 (2002).

11. T. Bollenbach, K. Kruse, P. Pantazis, M. González-Gaitán, F. Jülicher, Phys. Rev. Lett. 94, 018103 (2005).

12. M. Affolter, K. Basler, Nat. Rev. Genet. 8, 663 (2007).

13. M. Zecca, K. Basler, G. Struhl, Development 121, 2265 (1995)

14. T. Lecuit, W.J. Brook, M. Ng, M. Calleja, H. Sun, S.M. Cohen, Nature 381, 387 (1996); Nature 382, 93(E) (1996).

15. K. Basler, G. Struhl, Nature 368, 188 (1994).

16. B.A. Edgar, C.F. Lehner, Science 274, 1646 (1996).

17. P.A. Lawrence, G. Struhl, Cell 85, 951 (1996).

18. S.J. Day, P.A. Lawrence, Development 127, 2977 (2000).

19. D. Rogulja, K.D. Irvine, Cell 123, 449 (2005).

20. T. Aegerter-Wilmsen, C.M. Aegerter, E. Hafen, K. Basler, Mech. Dev. 124, 318 (2006).

21. L. Hufnagel, A.A. Teleman, H. Rouault, S.M. Cohen, B.I Shraiman, Proc. Natl. Acad. Sci. U.S.A. 104, 3835 (2007).

22. G. Schwank, S. Restrepo, K. Basler, Development 135, 4003 (2008).

23. Y. Hatwalne, S. Ramaswamy, M. Rao, R.A. Simha, Phys. Rev. Lett. 92, 118101 (2004).
24. K. Kruse, J.F. Joanny, F. Jülicher, J. Prost, Eur. Phys. J. E 16, 5 (2005).

25. J.F. Joanny, F. Jülicher, K. Kruse, J. Prost, New J. Phys. 9, 422 (2007).

26. A. Basu, J.F. Joanny, F. Jülicher, J. Prost, Eur. Phys. J. E 27, 149 (2008).

27. J.A. Glazier, F. Graner, Phys. Rev. E 47, 2128 (1993).

28. H.M. Byrne, J.R. King, D.L.S. McElwain, L. Preziosi, Appl. Math. Lett. 16, 567 (2003).

29. B.D. MacArthur, C.P. Please, Math. Biol. 49, 537 (2004).

30. B.I. Shraiman, Proc. Natl. Acad. Sci. U.S.A. 102, 3318 (2005).

31. D. Drasdo, Adv. Compl. Syst. 8, 319 (2005).

32. R. Farhadifar, J.-C. Röper, B. Algouy, S. Eaton, F. Jülicher, Curr. Biol. 17, 2095 (2007).

33. T. Bittig, O. Wartlick, A. Kicheva, M. González-Gaitán, F. Jülicher, New J. Phys. 10, 063001 (2008).

34. P.N. Adler, Dev. Cell 2, 525 (2002).

35. Y. Gong, C. Mo, S.E. Fraser, Nature 430, 689 (2004).

36. L.A. Baena-Lopez, A. Baonza, A. García-Bellido, Curr. Biol. 15, 1640 (2005).

37. A. García-Bellido, P. Ripoll, G. Morata, Nat. New Biol. 245, 251 (1973).

38. M. Théry, A. Jiménez-Dalmaroni, V. Racine, M. Bornens, F. Jülicher, Nature 447, 493 (2007).

39. E.V. Entchev, A. Schwabedissen, M. González-Gaitán, Cell 103, 981 (2000). 